\title{
Determination of Operational Efficiency in Urban Public Transport Lines
}

\author{
Ahmet ATALAY ${ }^{*}$, , Ömer Faruk BİRCiK ${ }^{2}$. \\ ${ }^{1}$ Assist. Prof. Dr. Civil Engineering Department, Engineering Faculty, Ataturk University, Erzurum, Turkey \\ ${ }^{2}$ Ms.C. Civil Engineer, Bingöl Provincial Directorate of Environment and Urbanization, Bingöl, Turkey.
}

\section{Keywords}

Public transportation,

Operational efficiency,

Clustering analysis,

$K$-means clustering,

Self-organizing mapping

\begin{abstract}
The increasing number of urban centers and the increasing number of vehicles caused by industrialization caused problems such as lack of infrastructure in traffic, environmental pollution and an increase in energy requirements. This situation led the city administrators to search for solutions in order to improve the efficiency of public transportation systems and increase their efficiency. In this study, it is aimed to determine the functional efficiency of the bus lines used in urban public transportation. For this purpose, the lines are classified according to their functional activities by using the functional data of the lines. Both classical cluster analysis and self-organizing mapping (SOM) method were used for classification. Data from Erzurum main public transport lines were used to implement the methods. According to the findings of this study, it was determined that the two methods achieved similar results. As a result, it has been determined that classification of public transportation lines used in cities according to their functional efficiency will be beneficial for decision makers to make correct planning. With the right planning in public transport lines, significant economic and environmental benefits will be obtained.
\end{abstract}

\section{Introduction}

With the rise in income levels in our cities, the intense demand has been towards private car use; transport investments have also been made to support the use of private automobiles. In this process, public transportation has always been in the background. This situation continued until the urban roads were filled with private cars and urban transportation reached a halt

Public transportation, which is the most important solution of transportation problems, increases its importance with the rapidly developing urbanization awareness and environmental sensitivity throughout the world.

An efficient public transport system; infrastructure, public transport, users of vehicles, operators and managers can be considered as the best way to take advantage of these components, can be achieved by creating a harmonious and integrated public transport system [1]

Providing the transportation needs of the people living in cities in terms of environment and economics is possible with public transportation systems. Public transport systems reduce the number of vehicles in traffic, prevent environmental and noise pollution and minimize fuel consumption. The performance of public transport systems, which have an important function both in terms of environment and economy, is of great importance in terms of meeting the public needs in a sustainable way.

The poor performance of public transport lines will result in lower service levels and will result in increased taxes and transportation fees to subsidize this economically unsustainable system. This will cause private vehicles that are not environmentally and economically efficient to be preferred over public transport.

The use of public transportation in cities is encouraged and studies are being made to increase its quality in order to make its use more attractive [2]. Evaluation of quality in public transport services has been carried out especially for the last fifteen years [3]. Performance determinations have become an important tool for transport providers aiming at setting strategic goals for the continuous improvement of the services provided [4]. Improvements in public transport services can affect user satisfaction in travel conditions and as a result, individuals' overall quality of life assessments [5].

There may be significant differences in the level of quality offered and what factors are crucial to the service, depending on the viewpoint (service managers 'point of view and passengers' point of view) to analyze service quality. Parkan (2002) argued that when the quality of service assessment was conducted by public transport suppliers, the list of factors that were considered to be important differed from the main factors considered by public transport users [6]. Because passengers have different perceptions about each line feature due to their special needs and even preferences.

When the studies in the literature are examined, an analysis of satisfaction with public transport services has been made [4], [5], [6]. Zhang et al., examined the effects of organizational forms on the comprehensive effectiveness of public transport services in China. In the study, comprehensive effectiveness was evaluated [7]. It used a combined evaluation method consisting of information entropy theory and data envelopment analysis. Abenoza et al., (2017) identified and characterized current and potential users of public transport in Sweden and identified the most important determinants of travel satisfaction with Public Transport services for each segment of travelers [8].

The classification of public transport lines by clustering analysis was carried out by Jimenez et al., (2013;2014) [9, 10]. Jimenez et al, (2013) developed a cluster method to examine and classify bus lines in Madrid. In this study, kinematic data of bus lines were collected that data on the movement of each bus such as; speed, distance, acceleration, deceleration, waiting time at the stop. In this study, the kinematic loops of the routes traveled by the vehicle are measured, the line loops are divided into micro loops and their characteristics are characterized, they are grouped into clusters with homogeneous kinematic properties in certain micro loops and a standard loop is created for each cluster. As a result, the hierarchical method with selection criteria, which can reduce the least negative combination of 
route groups, is preferred [9]. Jimenez et al, because it is costly and time-consuming to obtain kinematic variables, the classification of lines is made using macroscopic variables and artificial neural networks [10].

The most important problems for both users and operators in public transportation are loss of time and energy. In order to reduce these losses, especially to ensure energy efficiency in transportation, it should be ensured that the lines used in public transportation are used efficiently. In order to determine the efficient use of public transport lines, their performance should be determined. Evaluating the performance of the lines after they are determined can help in making the right decisions. In this study, it is aimed to classify public transportation lines according to their performance. The purpose of the classification is to identify lines with high performance and low performance. In this study, 33 bus lines belonging to Erzurum Metropolitan Municipality and Private Companies operating in Erzurum have been classified according to their performance. For classification, clustering analysis has been performed by using both traditional and artificial intelligence methods. Traditional methods, $\mathrm{k}$-means technique, artificial intelligence methods, self-organizing mapping (SOM) technique have been used. The results obtained with both methods have been compared.

\section{Materials and Methods}

The study material consists of data obtained from Erzurum Metropolitan Municipality Department of Transportation and Smart Card Directorate [11]. The data used in the study are described below.

\subsection{Capacity Utilization Rate (CUR)}

In Erzurum, there are buses of Metropolitan Municipality and public buses for public transportation. These buses have a seating capacity of 58 and 100 seats. Capacity utilization ratio (CUR) is calculated according to the following equation (1) by using the number of passengers, number of flights and bus capacities of the line.

$$
\text { CUR }=\frac{y}{n * k} * 100
$$

where;

y: Total number of passengers carried on line

k: bus capacity

$\mathrm{n}$ : number of bus travels

CUR values were calculated separately for both weekdays and weekends.

\subsection{Number of Passengers}

The average number of buses that buses carry in one day. This data was determined separately for both weekdays and weekends.

\subsection{Number of Passengers Moved at Peak Hour}

It is the average number of passengers carried at peak hours, morning and evening, on weekdays.

\subsection{Distance covered by buses}

It is the distance that the vehicles serving on each bus line travel on weekdays and weekend days.

The aim of the cluster analysis is to divide the units into groups according to their characteristic similarities. Standardization of the data before the similarities are calculated is important in confirming the results. In this study, the number of passengers, bus numbers and capacities on the line and so on. The numerical magnitudes of the values were found to be quite different from each other. The most common form of standardization is to convert the variables into standard values known as " $z$ scores". For this purpose, standard values were obtained for each variable using the equation (2).

$$
z=\frac{\left(x_{i}-\mu\right)}{\sigma}
$$

Z: Standardized value of each variable

xi: Raw value of each variable

$\mu$ : Average value of each variable

$\sigma:$ Standard deviation of each variable

In the inventory of Erzurum Metropolitan Municipality, there are 33 buses in total and 205 buses of two different types and capacities. In addition, there are 94 minibuses in six different lines used in intermediate public transportation. The lengths of the buses are quite different and vary from 15 to $55 \mathrm{~km}$. Buses with a capacity of 58 and 100 people, Dadaskent, Hilalkent, Ilıca, Istasyon, Ylldızkent and six different referral and administration centers are being routed.

Table 1. Referral and Administration Center (RAC) to which the lines are connected in Erzurum

\begin{tabular}{ll}
\hline $\begin{array}{l}\text { Referral and } \\
\text { Administration } \\
\text { Center (RAC) }\end{array}$ & Lines \\
\hline Ilıca & \\
Dadaskent & B1, B7 \\
Hilalkent & B2/A, B2, B3, B6 \\
Kayakyolu & G4, G5, G10 K7/A, K10 \\
Ylldzkent & G3, G6, G7, G7/A, K2,D1 \\
Istasyon & B4, B5, G1,K1,K8, G4/A, G8, K4, K5, K6, \\
& K9,B8,G2 \\
\hline
\end{tabular}

In statistical analysis, units with contradictory values among the analyzed units can change the results of the analysis. In this study, many scenarios have been formed with raw data and standard data in clustering analysis. It has been determined that the G9 line has been separated from the other lines as an outlier line. When the G9 line was excluded from the analysis, it was observed that the number of cluster elements was close to each other in the obtained clusters. This is because the G9 line's number of passengers, line length, vehicle number and capacity, capacity utilization rate etc. values are very different from the values of other lines. Therefore, it has a negative effect on the results of all analyzes. For this reason, G9 line was not used in the analyzes because of the outlier value.

\subsection{Clustering Analysis}

Clustering analysis, which is a multivariate statistical method, is used to classify data into subgroups by classifying them according to similarity rates [12]. The main purpose of this method is to group the variables based on their characteristics [13]. The cluster analysis developed by MacQueen in 1967 can be grouped under two headings as hierarchical and non-hierarchical clustering analysis, which is widely accepted and used [14], [15], [16].

Cluster analysis is an objective method developed to assess the structural characteristics of observations. In cluster analysis, researchers need to make a reliable sample selection that accurately represents the structure of the population. The success of cluster analysis depends on a good sample selection.

\section{6. k-means Clustering Method}

In this study, k-means clustering method and self-regulated mapping method which is one of the artificial intelligence clustering methods will be used.

In k-means clustering method, a new cluster center is created at each iteration and if an element is closer to the recalculated new center, it is moved to that cluster. 


\subsection{Determination the Number of Clusters}

Most clustering methods require determining the number of clusters that will occur at the end of the analysis. Especially in non-phased clustering methods, the number of clusters must be determined before starting the analysis. In progressive methods, such a determination is not required before analysis. With the help of the dendrogram obtained in progressive clustering methods, the number of clusters can be decided visually. For this reason, before applying non-staged clustering methods to the data, probable number of clusters can be determined by applying staged methods [17].

\subsection{Self-Organizing Mapping-SOM}

Self-Organizing Mapping (SOM), which is a more sophisticated and artificial neural network-based clustering technique than $\mathrm{K}$-means technique, has received considerable attention in a wide range of applications from trade to engineering [18], [19] [20], [21].

Self-editing maps are used to aggregate data and reduce the dimensionality of the data. According to some studies, the SOM method; shows the relationship between sets of data points in sets and sets in a two-dimensional space [18], [19], [20]. The SOM network can also display data in one or three-dimensional space. The SOM network consists of two neuron layers, the input and output layers (Figure 1).

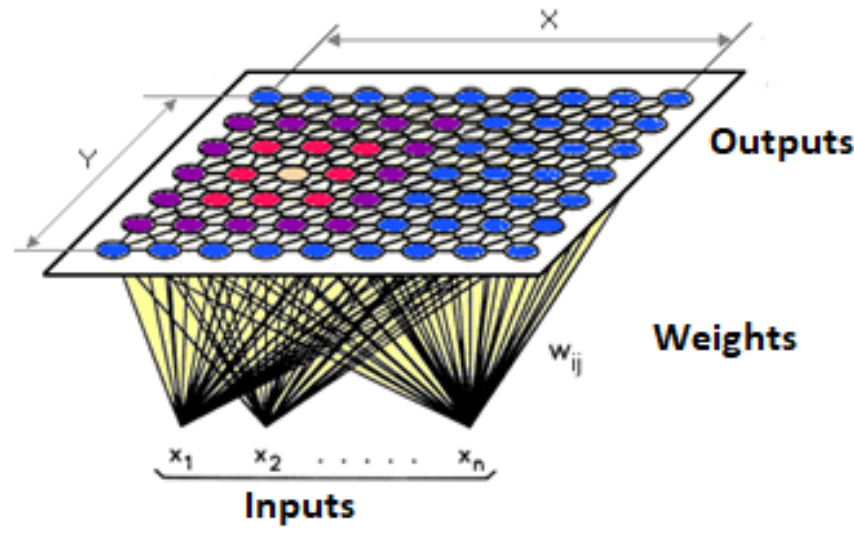

Figure 1. Example self-organizing map network structure

In the Self-Organizing Map (SOM) network, the input layer is fully connected to the output layer. The output layer, which is the core of the SOM network, works in a similar way to biological systems in that it suppresses sparse data representation and provides intensive data dissemination using a two-dimensional map. This can be done by assigning different sub-areas of the output layer to different categories of information. As a result, the position of the processing element in a network is assigned a certain characteristic in the input data set [22]. The network passes through several training cycles, starting with randomly selected weights for the nodes in the output layer, and is subjected to a self-regulation process. During each cycle, each input vector is considered corresponding and the winning node is found by equation (3), which gives the minimum distance

$\left\|x_{v}-v_{i}\right\|=\min \left\|x_{v}-v_{i}\right\|, i=1,2, \ldots, N$

In Equation (3), the symbol $\|$.$\| indicates the Euclidean distance$ commonly used to measure the distance between vectors. The weight vectors of the winning node and the weight vector of neighboring nodes are updated using equation (4).

$\Delta w=a\left(x_{v}-v_{i}^{\text {old }}\right)$, for $i \in N_{r}$

The input vector $x v$ in Equation 4 represents the learning coefficient $\alpha$ and the set of all nodes adjacent to $\mathrm{Nr} r$. The SOM clustering process works as follows ([23], [19]):

Step1: Weights start by selecting random small numbers (0-1)
Step2: The xv-input sample is placed in the SOM network and the distance between the weight vectors wj and the input sample $x v$ is calculated. Next, as in equation (5), the shortest neuron with $x i$ is selected. This selected neuron is called a 'winning' node.

Step3: Assuming a learning coefficient of $\alpha$, the weights of the winning node and its neighboring nodes are updated using the following equation.

$w_{j}^{\text {new }}=w_{j}+a\left(x_{v}-v_{i}^{\text {old }}\right)$, for $j \in N_{r}$

Step4: Step2 and Step3 are repeated until the stop criterion is met.

Intuitively, the output nodes that are close to the winning node receive more updates, and over time, the amount and coverage of the update decreases, and the link weights of the map nodes tend to stabilize. Neighborhood distance (r) may vary and generally decreases as training progresses [19]. The basic basis of the SOM network is described here. Various variations of SOM may also be found in the literature [18], [22], [24].

\section{Results}

In this study, it was checked whether the data in the database prepared for cluster analysis were meaningful or not. The variables not suitable for cluster analysis were excluded from the analysis. The variance analysis table of the variables used in the cluster analysis is obtained as in Table 2.

In this study, the clustering analysis made according to the values of the lines consists of the elements with the first set minimum, the second set middle, the third set maximum values from the distances between the result set centers. The second set and the third set are very similar. The first set and the third set are at least similar.

Clustering results according to SOM method were obtained with kmeans method. The SOM structure is constructed as shown in Figure 2. The ready toolbox in Matlab for the SOM network also generates an equal number of rows and columns. In this study, special code was written in Matlab to produce three outputs with SOM network. Accordingly, the structure of the network is formed with three inputs with ten inputs.

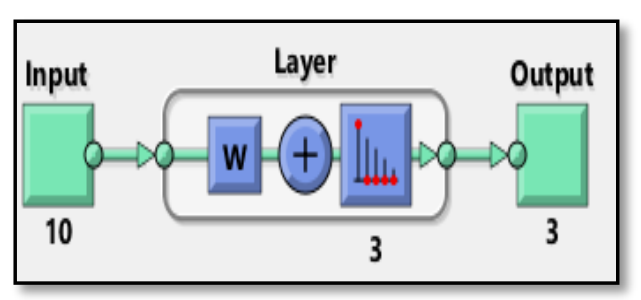

Figure 2. SOM network structure in this study

According to SOM method, clusters and element numbers and cluster values are obtained as in Figure 3. In this study, the number of clusters was determined as three by experiment.

In clustering analysis the number of clusters was decided after the suitability of the data had been determined. Using the data in this study, cluster analysis for experimental purposes was made. As a result of these trials, the number of clusters was determined as three. The distance values between the cluster result centers provide information about the similarity between the clusters (Table 3). 
Table 2. Variance analysis table

\begin{tabular}{|c|c|c|c|c|c|c|}
\hline & \multicolumn{2}{|c|}{ Cluster } & \multicolumn{2}{|c|}{ Error } & \multirow[b]{2}{*}{$\mathrm{F}$} & \multirow[b]{2}{*}{ Sig. } \\
\hline & $\begin{array}{l}\text { Mean } \\
\text { Square }\end{array}$ & $d f$ & $\begin{array}{l}\text { Mean } \\
\text { Square }\end{array}$ & $\mathrm{df}$ & & \\
\hline $\begin{array}{l}\text { Weekday } \\
\text { CUR }\end{array}$ & 7.327 & 2 & .481 & 29 & 15.231 & .000 \\
\hline $\begin{array}{l}\text { Weekend } \\
\text { CUR }\end{array}$ & 7.721 & 2 & .423 & 29 & 18.260 & .000 \\
\hline $\begin{array}{l}\text { Average } \\
\text { number of } \\
\text { passengers } \\
\text { per week }\end{array}$ & 9.428 & 2 & .096 & 29 & 98.438 & .000 \\
\hline $\begin{array}{l}\text { Average } \\
\text { number of } \\
\text { passengers } \\
\text { at the } \\
\text { weekend }\end{array}$ & 9.428 & 2 & .096 & 29 & 98.438 & .000 \\
\hline $\begin{array}{l}\text { Average } \\
\text { number of } \\
\text { passengers } \\
\text { per } \\
\text { morning } \\
\text { peak hour }\end{array}$ & 10.371 & 2 & .302 & 29 & 34.383 & .000 \\
\hline
\end{tabular}

\begin{tabular}{|c|c|c|c|c|c|c|}
\hline $\begin{array}{l}\text { Average } \\
\text { number of } \\
\text { passengers } \\
\text { per } \\
\text { evening } \\
\text { peak hour }\end{array}$ & 8.878 & 2 & .272 & 29 & 32.690 & .000 \\
\hline $\begin{array}{l}\text { Number of } \\
\text { vehicles } \\
\text { running } \\
\text { on } \\
\text { weekdays }\end{array}$ & 9.766 & 2 & .140 & 29 & 69.710 & .000 \\
\hline
\end{tabular}

\begin{tabular}{|c|c|c|c|c|c|c|}
\hline $\begin{array}{l}\text { Number of } \\
\text { vehicles } \\
\text { running } \\
\text { on the } \\
\text { weekend } \\
\text { line }\end{array}$ & 8.752 & 2 & .154 & 29 & 56.725 & .000 \\
\hline $\begin{array}{l}\text { Average } \\
\text { distance } \\
\text { traveled } \\
\text { during the } \\
\text { week }\end{array}$ & 11.535 & 2 & .085 & 29 & 135.530 & .000 \\
\hline $\begin{array}{l}\text { Average } \\
\text { distance } \\
\text { traveled at } \\
\text { the } \\
\text { weekend }\end{array}$ & 10.916 & 2 & .127 & 29 & 86.127 & .000 \\
\hline
\end{tabular}

Table 3. Distance between result cluster centers

\begin{tabular}{cccc}
\hline Cluster & 1 & 2 & 3 \\
\hline 1 & & 4.061 & 6.454 \\
2 & 4.061 & & 2.456 \\
3 & 6.454 & 2.456 & \\
\hline
\end{tabular}

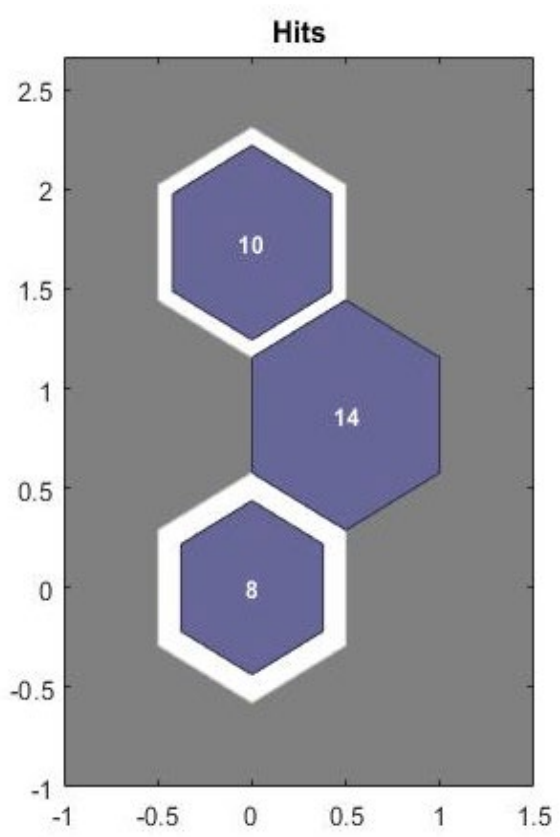

Figure 3. Clusters and number of cluster elements in SOM

The naming of the clusters is made according to the values of the clusters shown in Figure 3 as high, medium and small.

Table 4. The naming of clusters

\begin{tabular}{llc}
\multicolumn{3}{c}{ Table 4. The naming of clusters } \\
\hline & $\begin{array}{l}\text { Cluster No } \\
\text { (Cluster Name) }\end{array}$ & $\begin{array}{l}\text { Number of Elements in } \\
\text { Cluster }\end{array}$ \\
\hline Cluster & 1 (Minimum) & 8 \\
& 2 (Medium) & 14 \\
& 3 (Maximum) & 10 \\
\hline Total & & 32 \\
\hline
\end{tabular}

In this study, the same results were obtained according to two methods. The elements in each cluster are shown in Figure 4.

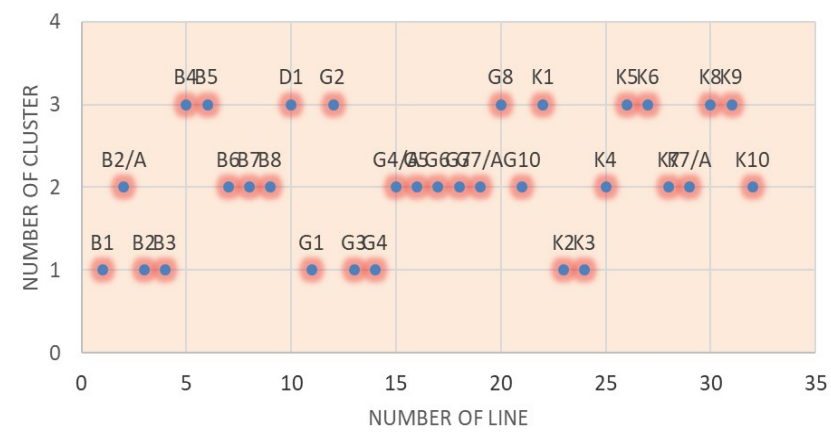

Figure 4. Distribution of bus lines by clusters

In some studies, in the literature, the operational efficiency of public transportation lines has been used in multi-criteria decision making and data envelope analysis. In these studies, the lines were ranked according to the scores determined [25], [26]. In our study, public transportation lines are divided into clusters according to their operational efficiency.

\section{Conclusion}

This study aimed to classify the lines according to their performance. Clustering analysis method was used to classify the lines. Two different methods were used in cluster analysis. The first method is $k$-means method and the second method is artificial intelligence, neural network clustering technique, self-regulated maps method is used. 
The same results were obtained according to the two methods used. There are 33 lines in Erzurum. It is not included in the analysis since it shows a difference in terms of line values G9 in comparison to other lines. The other 32 lines were divided into three clusters according to clustering analysis. The names of the clusters were made as big, medium and small.

The lines in the big cluster serve at full capacity and above. The lines in the middle cluster serve under capacity around the capacity. The lines in the small cluster serve under capacity.

It has been determined that the length of the lines used in public transportation service in Erzurum is approximately $45-50 \mathrm{~km}$. Larger line lengths increase both passenger and journey time.

As a result of the classification of public transportation lines, it was determined that they have a density above the capacity of the lines in the Istasyon RAC.

As a result of this study, while the classification of public transportation lines according to their performances, it was determined that $\mathrm{k}$-means technique and self-regulated maps techniques gave similar results. The classification results made on public transport lines can be a guide in re-planning line routes. The results of the classification will provide useful information to the decision-makers in the planning of urban transport, especially in the planning of public transport lines.

\section{Declaration of Conflict of Interests}

The authors declare that there is no conflict of interest. They have no known competing financial interests or personal relationships that could have appeared to influence the work reported in this paper.

\section{References}

[1.] Uludağ, N., Modeling of Bus Lines with Fuzzy Optimization and Linear Target Programming Approaches, in Institute of Science and Technology. (2010), Pamukkale University.

[2.] Paquette, J., et al., Measuring quality of service in dial-a-ride operations: the case of a Canadian city. Transportation, (2012). 39(3): p. 539-564

[3.] Redman, L., et al., Quality attributes of public transport that attract car users: A research review. Transport Policy, 2013. 25: $p$ 119-127.

[4.] Eboli, L., and Mazzulla, G.,, Service Quality Attributes Affecting Customer Satisfaction for Bus Transit. Journal of Public Transportation, 2007. 10(3): p. 21-34.

[5.] Ettema, D., et al., Satisfaction with travel and subjective wellbeing: Development and test of a measurement tool. Transportation Research Part F-Traffic Psychology and Behaviour, 2011. 14(3): p. 167-175.

[6.] Parkan, C., Measuring the operational performance of a public transit company. International Journal of Operations \& Production Management, 2002. 22(5-6): p. 693-720.

[7.] Zhang, C., Xiao, G., Liu, Y., Yu, F, The relationship between organizational forms and the comprehensive effectiveness for public transport services in China? Transportation Research Part A: Policy and Practice, 2018. 118: p. 783-802.

[8.] Abenoza, R.F., Cats, O., Susilo, Y.O, Travel satisfaction with public transport: Determinants, user classes, regional disparities and their evolution. Transportation Research Part A: Policy and Practice, 2017. 95: p. 64-84.

[9.] Jimenez, F., Román, A., López, JM, Methodology for kinematic cycle characterization of vehicles with fixed routes in urban areas. Transportation Research Part D, 2013. 22: p. 14-22.
[10.] Jimenez, F., Serradilla, F., Roman, A., and Jose, R., Naranjo. E, Bus Line Classification Using Neural Networks. Transportation Research Part D, 2014. 30: p. 32-37.

[11.] Bircik, Ö.F., Investigation of Erzurum Urban Public Transportation by Cluster Analysis Method. 2019, Ataturk University. p. 97.

[12.] Firat, M., Dikbaş, F., Koç, A. C., \& Güngör, M., Classification of Annual Precipitation and Determination of Homogeneous Regions by K-Means Method. Technical Journal, 2012. 383: p. 6037-6050.

[13.] Uslu, A., Çetinkaya, C., Özceylan,E., Işleyen, S.K., Analysis of Organized Industrial Zones by Hierarchical-K-Means Method. Turkish Journal of Social Sciences Research, 2017. 2(1): p. 20-37.

[14.] Atalay, A., \& Tortum, A., Cluster Analysis by the Provincial Traffic Accident in Turkey between the years of 1997-2006. Pamukkale University Journal of Engineering Sciences, 2010. 16(3): p. 335345 .

[15.] Tatlıdil, H., Applied Multivariate Statistical Analysis. 1996, Cem Ofset Ltd. Comp.

[16.] Ozdamar, K., Statistical Data Analysis with Multivariate Programs (Multivariate Analysis). 2002: Kaan Bookstore.

[17.] Punj, G. and D.W. Stewart, Cluster-Analysis in MarketingResearch - Review and Suggestions for Application. Journal of Marketing Research, 1983. 20(2): p. 134-148.

[18.] Demirhan, A.v.G., I, Image segmentation using self-organizing maps and gray level cooccurrence matrices. Journal of the Faculty of Engineering and Architecture of Gazi University, 2010. 25(2): p. 285-291.

[19.] Alpdogan, Y.a.B., H.S, Classification of course contents by using self-organizing maps. Journal of the Faculty of Engineering and Architecture of Gazi University, 2009. 24(2).

[20.] Breining, C., State detection for hands-free telephone sets by means of fuzzy LVQ and SOM. Signal Processing, 2000. 80(7): p. 1361-1372.

[21.] Yao, K.C., et al., Unsupervised segmentation using a selforganizing map and a noise model estimation in sonar imagery. Pattern Recognition, 2000. 33(9): p. 1575-1584.

[22.] Kiang, M.Y., M.Y. Hu, and D.M. Fisher, An extended selforganizing map network for market segmentation - a telecommunication example. Decision Support Systems, 2006. 42(1): p. 36-47.

[23.] Kim, K.J. and H. Ahn, A recommender system using GA K-means clustering in an online shopping market. Expert Systems with Applications, 2008. 34(2): p. 1200-1209.

[24.] Ince, H., Imamoglu, S.Z., Keskin, H.,, Comparison of SelfRegulated Map Networks and K-means Cluster Analysis: An Example of Consumer Profiling. Journal of the Faculty of Engineering and Architecture of Gazi University, 2013. 28(4): p. 723-731.

[25.] Güner, S. Operational Efficiency and Service Quality Analysis in Public Transportation Systems. Journal of Transportation and Logistics, 2017. 2(2): p.33-48.

[26.] Güner, S., Taşkın, K., Gürler G., Measurement of Service Efficiency of Local Public Transport Lines with DEA. Journal of Business Science (JOBS), 2017; 5(3): p. 127-145.

\section{How to Cite This Article}

Atalay, A., Biricik, Ö.F., Determination of Operational Efficiency in Urban Public Transport Lines, Civil Engineering Beyond Limits, 1(2021), 16-20. https://doi.org/10.36937/cebel.2021.001.004. 